BNL - 67638

\title{
SINGLE-PASS HIGH-GAIN FREE ELECTRON LASER ELECTRON BEAM DIAGNOSTICS
}

\author{
X.J. Wang \\ National Synchrotron Light Source \\ Brookhaven National Laboratory \\ Upton, New York 11973
}

May 2000

\section{National Synchrotron Light Source}

\author{
Brookhaven National Laboratory \\ Operated by \\ Brookhaven Science Associates \\ Upton, NY 11973
}

Under Contract with the United States Department of Energy

Contract Number DE-AC02-98CH10886 


\section{DISCLAIMER}

This report was prepared as an account of work sponsored by an agency of the United States Government. Neither the United States Government nor any agency thereof, nor any of their employees, nor any of their contractors, subcontractors or their employees, makes any warranty, express or implied, or assumes any legal liability or responsibility for the accuracy, completeness, or any third party's use or the results of such use of any information, apparatus, product, or process disclosed, or represents that its use would not infringe privately owned rights. Reference herein to any specific commercial product, process, or service by trade name, trademark, manufacturer, or otherwise, does not necessary constitute or imply its endorsement, recommendation, or favoring by the United States Government or any agency thereof or its contractors or subcontractors. The views and opinions of authors expresses herein do not necessarily state to reflect those of the United States Government or any agency thereof. 


\title{
Single-Pass High-Gain Free Electron Laser Electron Beam Diagnostics \\ X.J. Wang \\ Accelerator Test Facility, NSLS \\ Brookhaven National Laboratory, Upton, NY 11973
}

\begin{abstract}
Consensus reached in the last few years that fourth generation light source will most likely be a X-ray or a UV coherent source based on single-pass high-gain free electron laser (FEL), such as Self Amplified Spontaneous Emission (SASE), or seeded high-gain harmonic -generation (HGHG) free electron lasers. High-gain $\left(>10^{7}\right)$ required for single-pass FEL puts great constrain on the quality of electron beam, and demands detailed distribution information of electron beam in six -dimension. The typical accelerator system for single -pass FEL consists of a photocathode RF gun injection system, a linac and magnetic bunch compressors, and a long undulator. The major challenges in beam diagnostics for single-pass FEL are to characterize the pico-seconds high-brightness electron beam in six-dimension produced by photocathode RF gun injector, and impro ve the stability and reliability of the photocathode RF gun injection system. Characterization of short electron bunch ( $100 \mathrm{fs})$ produced by the compressors, and co-align the electron beam with FEL radiation inside long undulator are also critical for FEL performance. We will discuss many diagnostic techniques developed at the Brookhaven Accelerator Test Facility (ATF) for the photocathode RF gun injection system, present several techniques for femto-seconds bunch length measurement, especially RF kicker cavity. Tomography technique for both transverse and longitudinal phase space measurements is discussed. Concept of multiple alignment -laser stations is present for beam alignment in the long undulator.
\end{abstract}

\section{INTRODUCTION}

Since the discovery of $\mathrm{X}$-rays more than 100 years ago, improvement of the brightness of X-ray is largely due to the accelerator technology and synchrotron radiation (Figure 1), which in turn leads to progress and improvements in many science and technologies. The history of $8 \mathrm{keV}$ (Figure 1) shows that, X-ray brightness improvements mainly came from two fronts of accelerator technologies. First, and most import one, is the brightness of electron beam, which is best illustrated by the development of low emittance storage ring for the third generation light source. The other one is the radiation generation techniques, such as undulator and wiggler. The next generation light source must be able to provide improvement of the brightness of X-ray by ten orders of magnitude, and factor of ten or more reduction in radiation pulse length. To achieve the brightness and coherence of the X-ray for next generation light source, coherent radiation from Free electron laser (FEL) with small emittance $(<\lambda / 4 \pi$, where $\lambda$ is $X$-ray wavelength) and shorter electron beam $(<100 \mathrm{fs})$ is required. The energy scaling of the emittance and instabilities in the storage ring narrowed the choice of the future light source to the linac based UV or X-ray FEL.

FEL is capable of operating in two modes (Fig.2), most linac bas ed long wavelength FEL and storage ring FEL operating in the oscillator mode. Due to the challenges in making high quality mirrors in UV and $\mathrm{X}$-ray region, future $\mathrm{X}$-ray FEL will most likely

\footnotetext{
* Invited talk at BIW' 2000, work supported by the US DOE under contract DE-AC02-98CH10886.
} 
operate in single-pass, high-gain regime. Further more, single-pass FEL can operate either in a laser seeded manner, such as high -gain harmonic generation (HGHG)[1], or self-amplified spontaneous emission (SASE) [2].

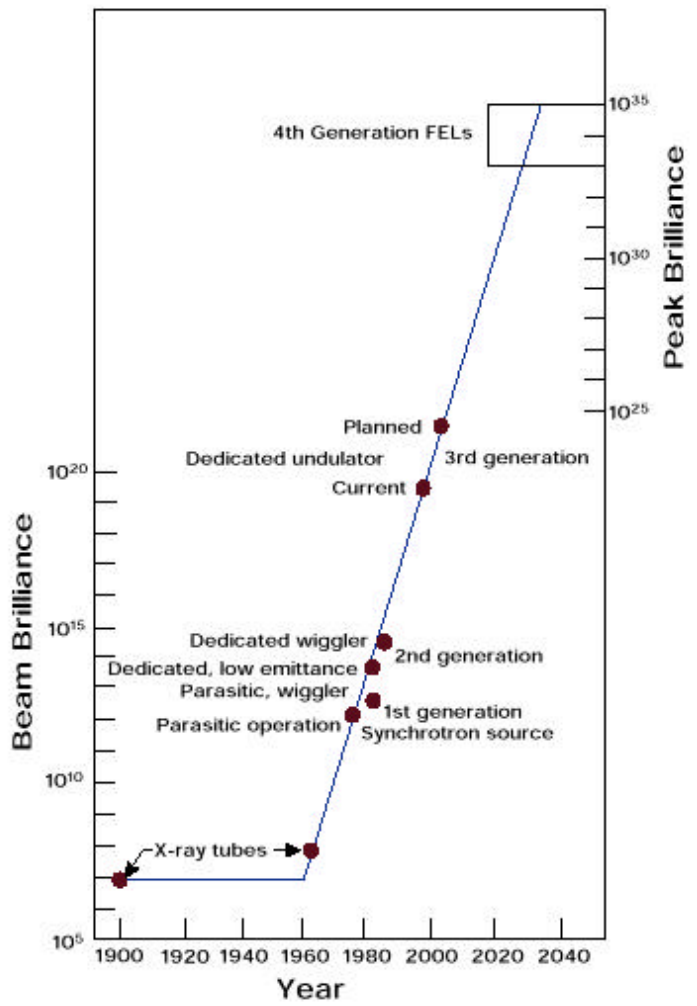

Fig.1 8 keV X-ray history
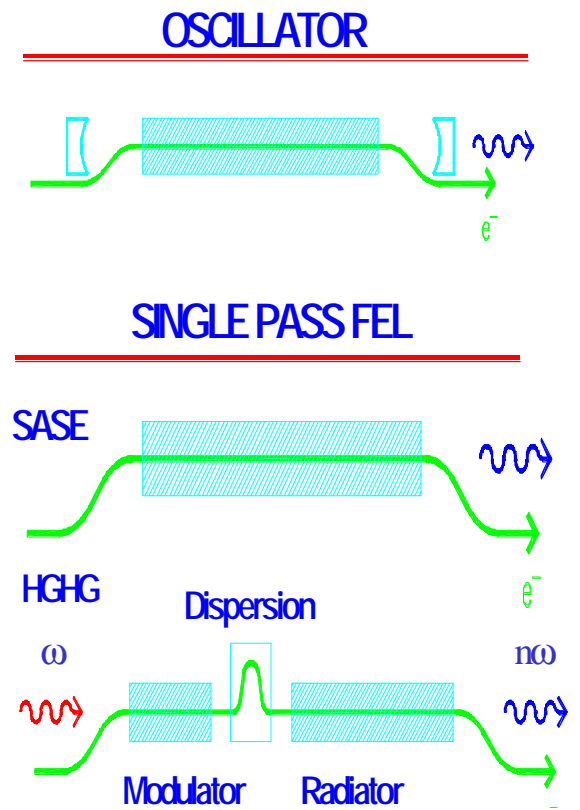

Fig.2 FEL Operating modes.

Single-pass high-gain free electron laser projects were proposed by many laboratories around the world to study the FEL physics, and demonstrate the potential future applications. The wavelength of those projects covers wide range. In the visible and

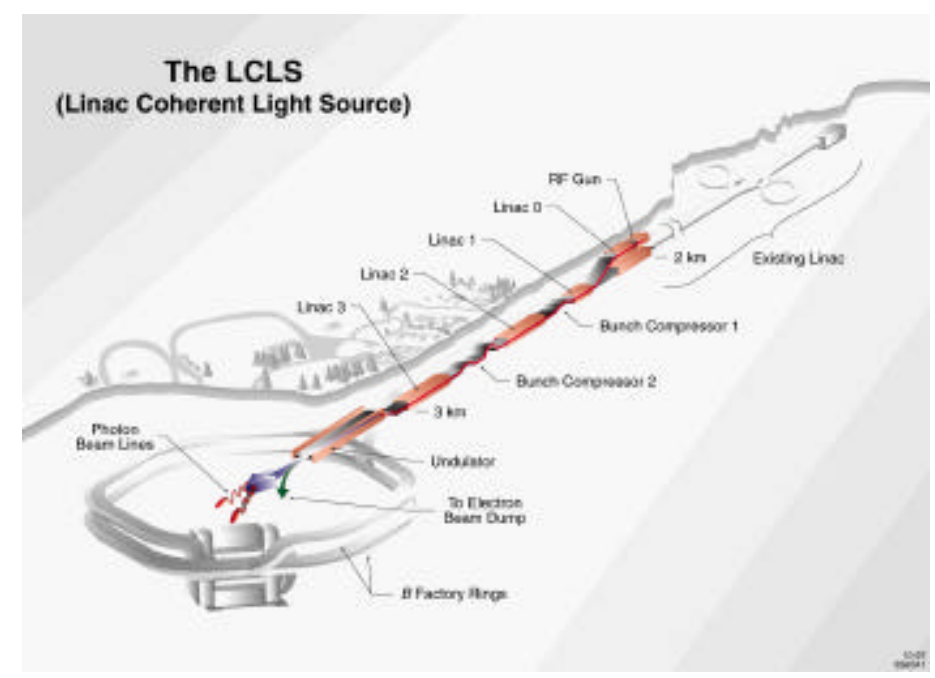

Fig.3 The $1.5 \approx X$-ray SASE FEL layout at SALC. 
UV region, VISA experiment [3] at the BNL accelerator Test Facility (ATF),

LEUTL [4] at APS of ANL, and TTF-FEL at DESY [5] are designed to study the SASE physics process in the visible to UV; while BNL DUV FEL [6] is designed to demonstrate HGHG at DUV and its applications. A $1.5 \approx \mathrm{X}$-ray FEL, Linear Coherent Light Source (LCLS) is under design studied by a SLAC led collaboration [7] (fig.3).Table I summarizes the major properties of those FEL projects. The parameters will have maj or impact on beam diagnostics are electron beam pulse length, spot size, undulator gap, undulator length, and radiation wavelength.

Table I: Summary of Single -Pass FEL projects

\begin{tabular}{|c|c|c|c|c|c|c|c|}
\hline & VISA & $\begin{array}{l}\text { BNL- } \\
\text { DUV } \\
\text { FEL }\end{array}$ & $\begin{array}{c}\text { BNL - } \\
\text { DUV FEL+ }\end{array}$ & $\begin{array}{c}\text { APS } \\
\text { LEUTL1 }\end{array}$ & $\begin{array}{c}\text { APS } \\
\text { LETUL2 }\end{array}$ & LCLS & $\begin{array}{l}\text { TTF } \\
\text { VUV } \\
\text { FEL }\end{array}$ \\
\hline $\begin{array}{l}\text { Energy } \\
(\mathrm{MeV})\end{array}$ & $\begin{array}{l}71- \\
100\end{array}$ & 210 & 310 & 217 & 700 & $\begin{array}{l}5000 \text { - } \\
15000\end{array}$ & $\begin{array}{l}390 \text { - } \\
1000\end{array}$ \\
\hline $\begin{array}{c}\text { Peak } \\
\text { current }(\mathbf{A})\end{array}$ & 200 & 1500 & 1500 & 100 & 500 & 3400 & $\begin{array}{l}500- \\
2500\end{array}$ \\
\hline $\begin{array}{c}\text { Emittance } \\
\text { (mm-mrad, } \\
\text { rms) }\end{array}$ & $2-3$ & 5 & 5 & 5 & 3 & 1.5 & 2.0 \\
\hline $\begin{array}{c}\text { Beta- } \\
\text { function } \\
\text { (m) }\end{array}$ & 0.3 & $3-5$ & $3-5$ & 5 & 5 & $15-20$ & $1-3$ \\
\hline $\begin{array}{l}\text { Spot size } \\
\text { (um, rms) }\end{array}$ & 60 & 200 & 100 & 150 & 100 & 30 & 55 \\
\hline $\begin{array}{c}\text { Radiation } \\
\text { wavelength } \\
(\mathbf{n m})\end{array}$ & $\begin{array}{c}800- \\
600\end{array}$ & $\begin{array}{l}200- \\
150\end{array}$ & $200-50$ & 530 & 50 & 0.15 & 42- 6 \\
\hline $\begin{array}{l}\text { Undulator } \\
\text { period }(\mathrm{cm})\end{array}$ & 1.8 & 3.9 & 1.8 & 3.3 & 3.3 & 3 & 2.73 \\
\hline $\begin{array}{l}\text { Undulator } \\
\text { gap (mm) }\end{array}$ & 6 & 22 & 6 & 9 & 9 & 6 & 12 \\
\hline $\begin{array}{l}\text { Undulator } \\
\text { length (m) }\end{array}$ & 4 & 10 & 6 & $9 \times 2.4$ & $10 \times 2.4$ & $20 \times 5$ & $15-30$ \\
\hline $\begin{array}{c}\text { Drift } \\
\operatorname{section}(\mathrm{cm})\end{array}$ & $\mathbf{0}$ & $\mathbf{0}$ & $\mathbf{0}$ & 23 & 23 & 27 & 32 \\
\hline Focusing & FODO & wire & FODO & external & external & FODO & FODO \\
\hline
\end{tabular}

Fig. 3 shows typical single -pass high-gain FEL. There are three key components. They are photocathode RF gun injection system; linac, beam transport and electron beam bunch compression system; and a long u ndulator to generate coherent radiation. The performance of a single -pass high-gain FEL is characterized by the gain length $\mathrm{L}_{\mathrm{G} 0}(1-\mathrm{D})$,

$$
L_{G 0}=\frac{\lambda_{u}}{4 \sqrt{3} \pi \rho}
$$

Where $\lambda_{u}$ is the undulator period, and $\rho$ is the Pierce parameter, which is determined by electron beam properties,

$$
\rho=\frac{1}{2 \gamma}\left[\frac{I}{I_{A}}\left(\frac{\lambda_{u} K[J J]}{\sqrt{2} 2 \pi \sigma_{x}}\right)^{2}\right]^{1 / 3}
$$


Where $\gamma$ is the beam energy measured in electron rest energy, $I$ is the electron beam peak current, $I_{A}=\mathrm{ec} / \mathrm{r}_{\mathrm{e}}=17045 \mathrm{~A}$ is Alfven current, $K=0.94 B \lambda_{u}$ is the undulator parameter, $\sigma_{x}$ is the rms electron beam spot size, $[\mathrm{JJ}]$ is the combination of Bessel functions. The FEL radiation wavelength is determined by,

$$
\lambda=\frac{\lambda_{u}}{2 \gamma^{2}}\left(1+\frac{K^{2}}{2}+\gamma^{2} \theta^{2}\right)
$$

Above equations show that, both peak current $I$ and spot size $\sigma_{x}$ could have significant impact on the gain of the single -pass FEL. That is, a good quality electron beam in both transverse and longitudin al phase space is needed for single -pass high gain FEL. Further more, the relative energy spread of the electron beam must be small comparing to $\rho$ when 3-D effects are considered. To produce and preserve the high brightness electron demanded by the single -pass high-gain FEL, electron beam diagnostics is the key. Many papers and working groups have been devoted to various aspects of FEL beam diagnostics [8-10], we will present latest development of electron beam diagnostics for single-pass high-gain free electron laser, particularly the techniques developed at the BNL ATF. In the following section, beam diagnostics for photocathode RF gun injection system is presented; the laser system diagnostics and its impact on the FEL performance are emphasized. Then the beam diagnostics requirements for linac and bunch compression system will discussed, we will discuss short bunch measurement requirement for single-pass high-gain FEL and bunch length measurement techniques, specially slice emittance, RF kicker cavity a nd tomography techniques. The beam alignments and diagnostics for the long undulator will be studied in the last section of this report; emittance measurement techniques and laser alignment will be also discussed in detail.

\section{BEAM DIAGNOSTICS FOR PHOTOCATHODE RF GUN INJECTION SYSTEM}

Beam diagnostics requirements for single -pass high-gain FEL is very similar to that for the future $\mathrm{e}^{+} \mathrm{e}^{-}$linear colliders. There are many differences. One of the most important difference is electron beam source and its impa cts on the beam diagnostics. Photocathode RF gun [11] is the only technique now capable of producing high -brightness electron beam for single-pass high-gain FEL. Fig.4 is the schematic of the BNL ATF photocathode RF gun injection system, consisting of sy nchronized laser system, photocathode RF gun, emittance compensation solenoid magnet, beam diagnostics station and an energy booster linac.

Whenever beam diagnostics for photocathode RF gun based linac system is discussed, the phase-locked RF gun las er system must be considered first. It is not only used to produce photoelectron beam, it can also be use to for beam diagnostics and studying laser and electron beam interactions. One of the challenges of the operating photocathode RF gun [12] is to provide stable and reliable laser beam. A good, high quality laser beam diagnostics system is absolute required for single -pass high-gain FEL based on the photocathode RF gun injection system. Laser system can be further divided into three components. They are the laser system on the table, including the oscillator and amplifiers; laser beam transport system, and beam delivery system to the RF gun (front end optics). 


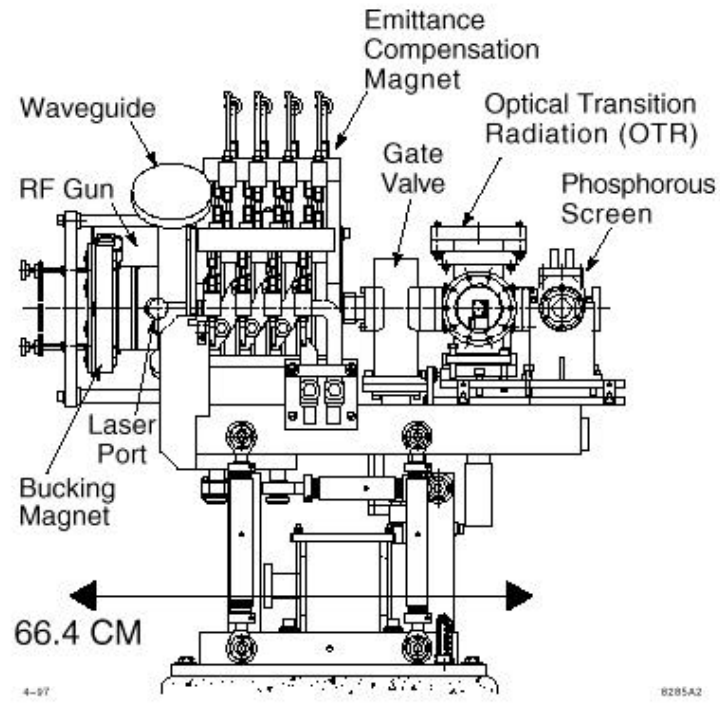

Fig. 4 The ATF photocathode RF gun injection system layout

The stability and reliabili ty of the mode locked oscillator is most critical for the overall system stability, the timing jitter and point stability of the oscillator must be monitored continuously. The laser mode quality and energy fluctuation should be monitored at each stage of amplifications. The major issues in laser beam transport system are air current and dust caused point instability and beam quality degradation. Laser beam diagnostics at the front end must be part of overall design of the photocathode RF gun injection system. There are two types of optical design used, one is laser beam normal incident on the cathode, other is oblique incident on the cathode. Oblique incident has many advantages in laser beam diagnostics. There will be no optics inside the vacuum, and it is easier to split small faction of laser beam for on -line monitoring. Front optics should measure the laser energy delivered to the cathode in real time; it should also provide an equivalent image of the laser on the cathode to monitor the laser point $s$ tability and spot size. Figure 5 is the front end optics of the ATF photocathode RF gun driving laser system, laser beam energy, beam profile and point stability are monitored continuously.

One of the most important features of the BNL ATF photocathode RF gun injection system design is the beam diagnostic station after the emittance compensation solenoid magnet (Fig.4). There are two cubes for beam diagnostics, the first one could also be used for normal incident of laser beam on the cathode. An aluminu m mirror installed inside the first cube now at ATF for laser beam alignment on the cathode, and optical transition radiation (OTR) based beam energy measurement. The second cube contains a combination of phosphor screen based beam profile monitor (BPM) and Faraday cage (Fig.6), which can provide all basic information needed for operating the photocathode RF gun injection system. The BPM screen is formed by depositing phosphor on a thin aluminum foil, the 45 œmirror behind the phosphor screen was polish ed by a solid Tustang which is designed to stop $8 \mathrm{MeV}$ electron beam. One of the important functions of metal screen is to intercept secondary electrons to insure the charge measurement accuracy of the Faraday cage. Using the steering coil upstream of the BPM, the photoelectron beam energy can be measured (Fig.7). 


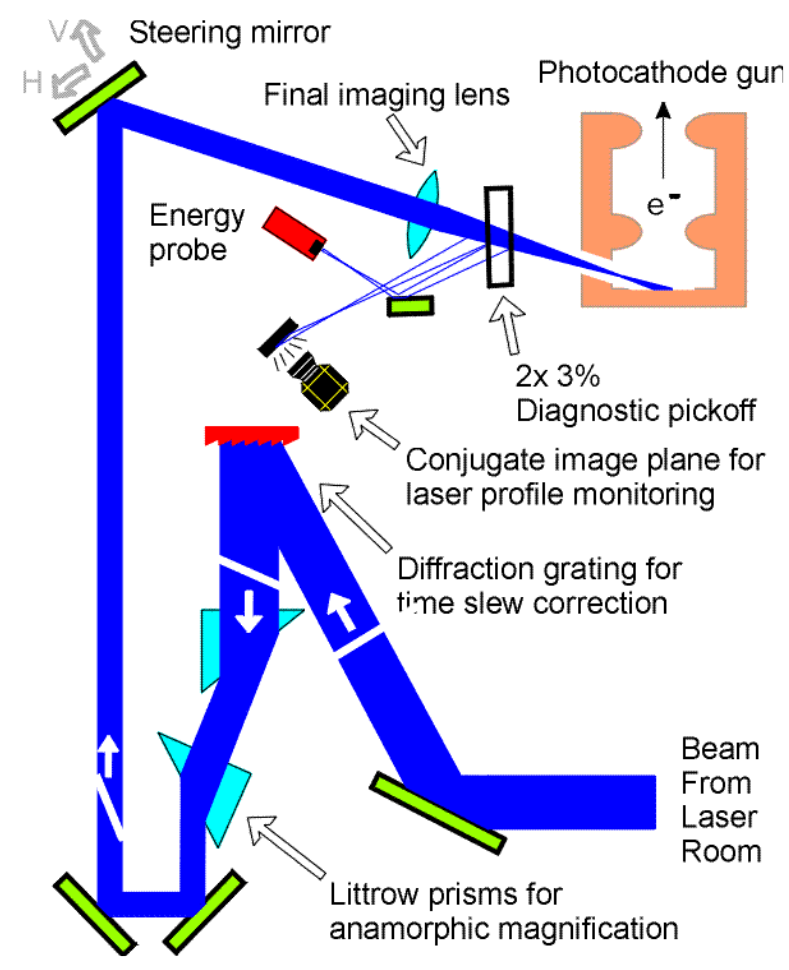

Fig.5 The ATF front-end optics for the RF gun injection system.

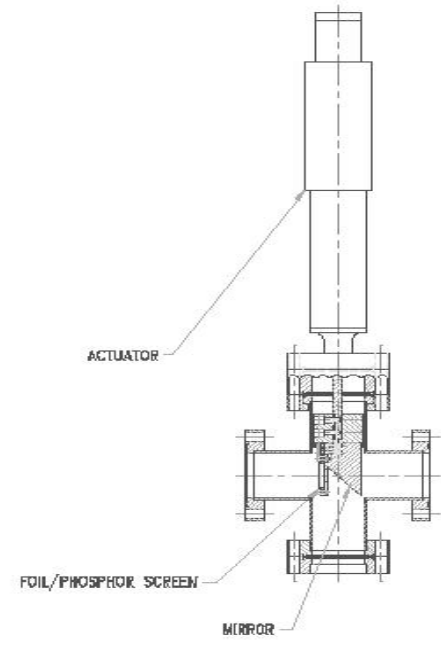

Fig.6 The ATF BPM and Faraday cage combination design.

The relative phase between RF field and laser pulse arrives on the cathode determine the qualities of photo-electron beam, both transverse emittance and electron beam pulse length are determined by the RF phase of laser arrives time. The RF phase can be determined by the measurement of photo-electron beam charge using the Faraday cage while varying the RF gun phase. The photoelectron beam current density $j$ is,

$$
j=A I(t)(h v-\varphi+\alpha \sqrt{\beta E(t)})^{2}
$$

Where $I(t)$ is the time dependent laser intensity, $h v$ is the photon energy, $\varphi$ is the cathode work function, $E(t)$ is RF field, and $\beta$ is the field enhancement factor. Since the photon 


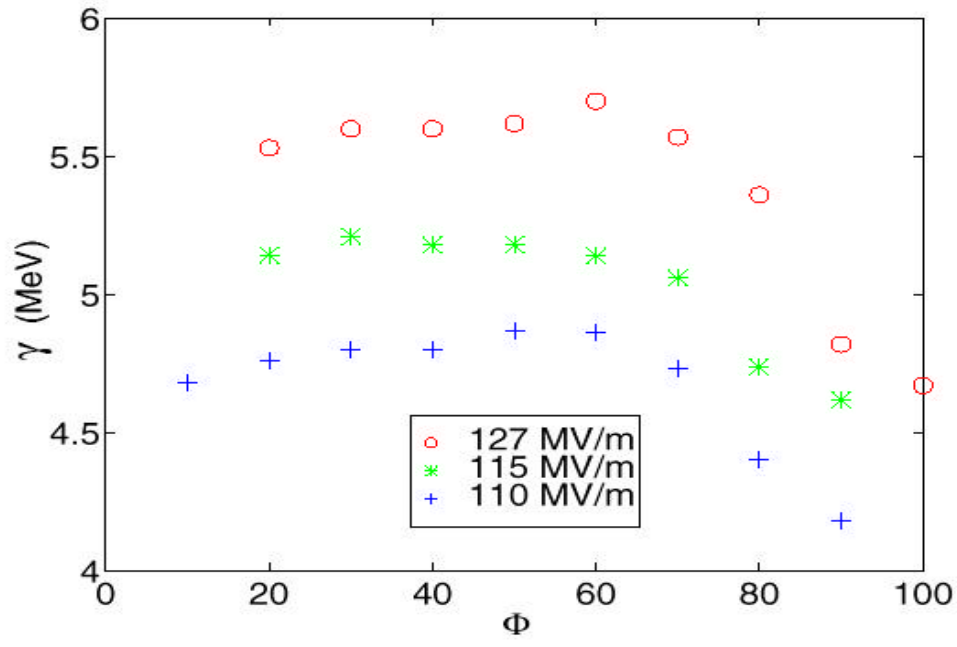

Fig.7 Photoelectron beam energy as function of the RF gun phase.

energy and cathode work function is very close for most metal cathode now used the photocathode RF gun, the emission process is dominated by the RF field, i.e., Schottky effect. The charge measured can be written,

$$
Q(\phi)=\int_{-\infty}^{\infty} d \tau A I(\tau)(h v-\varphi+\alpha \sqrt{\beta E(\phi-\tau)})^{2}
$$

The above equation shows that, the charge $Q(\phi)$ is the convolution of the laser intensity distribution $I(\tau)$ and RF field $E(\phi)$. So in principle, we could deduce the laser longitudinal distribution by measuring the photoelectron charge by de -convolution.

Fig.8 is the experimental measurement of charge as function of RF gun phase for $\mathrm{Mg}$ cathodes. Following information could be obtained fro m Fig.8,

- Absolution RF gun phase: For Schottky effect dominated photoemission, the peak charge $Q(\phi)$ corresponding to the RF field crest $(90 \mathrm{deg})$. This can be further calibrated at the zero RF phase.

- Timing Jitter: taking advantage of $Q(\phi)$ dependency on the RF gun phase, we can measure the timing jitter between the RF system and RF gun driving laser. Notice the asymmetry in Fig.8, the falling edge of the $Q(\phi)$ curve is much more sensitive to the laser timing jitter. For a maximum charge of $1.5 \mathrm{nC}$, the sensitivity of the measurement can be $35 \mathrm{pC} / \mathrm{deg}$. For a $\mathrm{S}$-band RF gun, one degree $\mathrm{RF}$ phase is almost equal to $1 \mathrm{ps}$, this easily allows us to measure femto -seconds timing jitter between the laser and RF system if laser energy fluctuation can be simultaneously tak e into consideration. To obtain the laser system timing jitter, the timing jitter dependency on the RF gun phase must be taken into consideration.

Another important technique for photoelectron beam diagnostics is laser mask. Fig.9 shows both laser and electron beam profiles, one of the important features is that, the rotation of electron beam profile relative to laser by the emittance compensation solenoid magnet. This feature can be used to identify laser point stabilities and laser profile distribution. Fig. 10 shows electron beam image of the laser mask on the BPM right after the solenoid magnet. By imaging electron beam of the laser mask on the BPM, we can identify the asymmetry in laser beam, non -uniformity of quantum efficiency on the cathode, and the location of the laser on the cathode. 


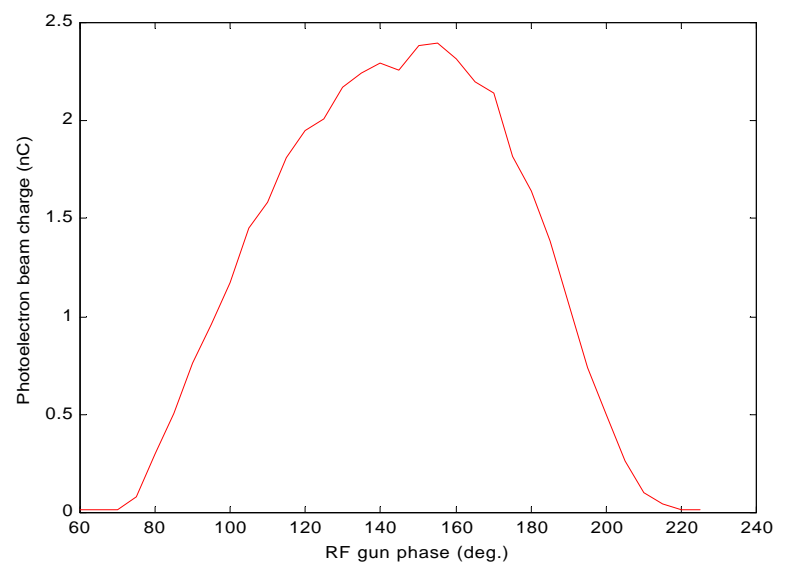

Fig.8 Photoelectron charge as a function of RF gun phase.

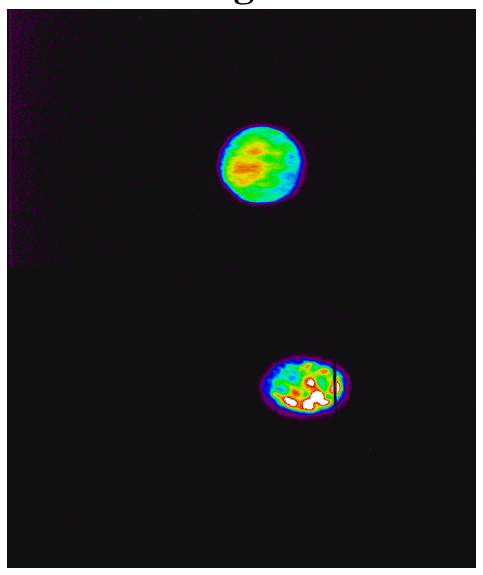

Fig.9 laser (top) and electron beam (bottom) images.

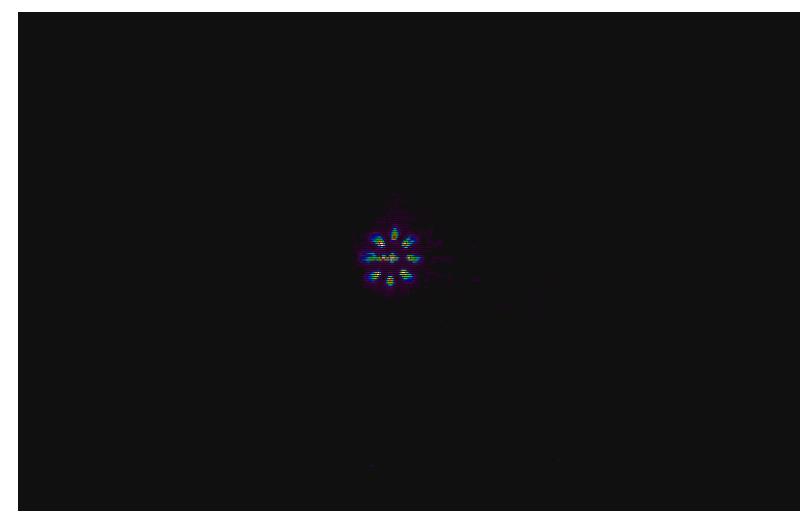

Fig.10 Electron beam image of laser mask.

Timing jitter of the photoelectron beam not only cause peak current jitter during the bunch compression, it also introduces charge variation due to the Schottky effect. By mixing the sum signal of the stripline beam position monitor with $S$-band RF reference signal (Fig. 11) [13], the timing jitter of the photoelectron beam can be characterized. The resolution of the timing jitter better than 200 fs was realized because timing jitter of the laser will introduce both intensity (the Schottky effect) and relat ive phase change between the reference signal and photoelectron beam.

The most important beam diagnostics for energy booster linac is the beam profile monitor (BPM) after the second section of the boost linac at the energy about $50 \mathrm{MeV}$. This BPM is used to tune up electron beam and minimize the emittance. By measuring the beam profile asymmetry, the misalignment of the photoelectron beam relative to the linac structure can be reduced. The emittance minimization during the emittance compensation process is control by the solenoid magnet, minimize the beam spot size on the BPM is the starting point for this process. 


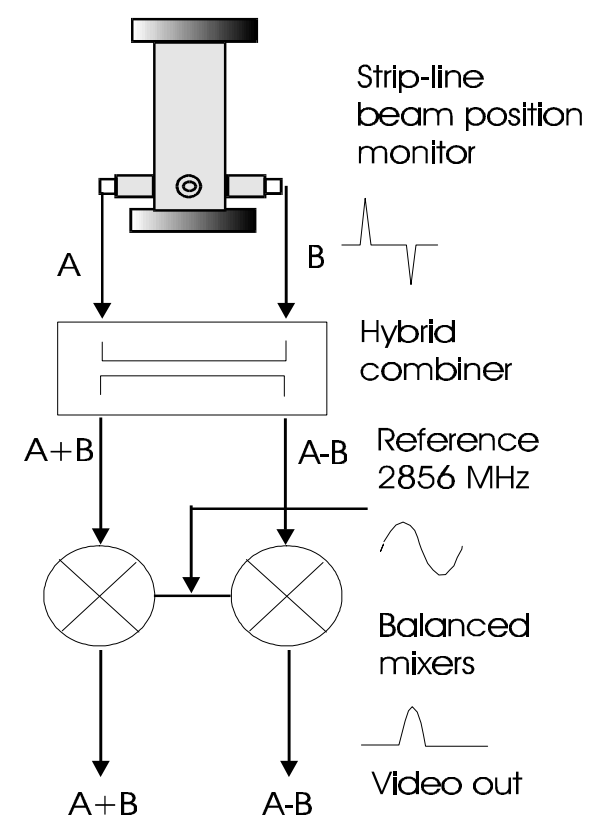

Fig. 11 Timing jitter measurement schematic.

\section{SHORT ELECTRON BUNCH CHARACTERIZATION}

To achieve the peak current required for the single-pass high-gain FEL, pico-seconds electron beam produced by the RF gun must be further compressed down to the order of $100 \mathrm{fs}$. One of the critical issues during the bunch compression is the emittance growth caused by the Coherent Synchrotron Radiation (CSR) and Centrifugal Space Charge Force (CSCF)[14]. Recent theoretical studies indicated that, electron beam distribution in both transverse and longitudinal dimension could have significant effect on CSR and $\mathrm{CSCF}$ [15]. One of the basic physical process for single -pass high-gain FEL is interaction between the electron beam with the electromagnetic radiation fields. This interaction leads to electron beam micro-bunching on the order of the radiation wavelength, and electrons within the so-called coherent-length radiate coherently. In summary, to understand the single-pass high-gain FEL and emittance growth during the compression, bunch length measuring technique capable of measuring the electron beam distribution within the cohere nt length is required.

Tremendous progress has been made in sub pico -second bunch length measurement techniques in the past decade $[8,10]$. Broad range of short bunch measurement technique was investigated, from time domain to frequency domain, such a s streak camera, fluctuation spectrum; and coherent radiation.

Streak camera is the technique for time domain measurement. It has many attractive features, such as single shot and capable of synchronized with the RF system. The best demonstrated resolution (Fig.12) is about $370 \mathrm{fs}$ [16]. The disadvantages of using streak camera for short bunch electron beam measurement are space -charge limited dynamic range, resolution limitation, and broad -band radiation generated by the short electron beam. Streak camera could play an important role in photocathode RF gun laser system diagnostics and electron beam diagnostics before the bunch compression. 

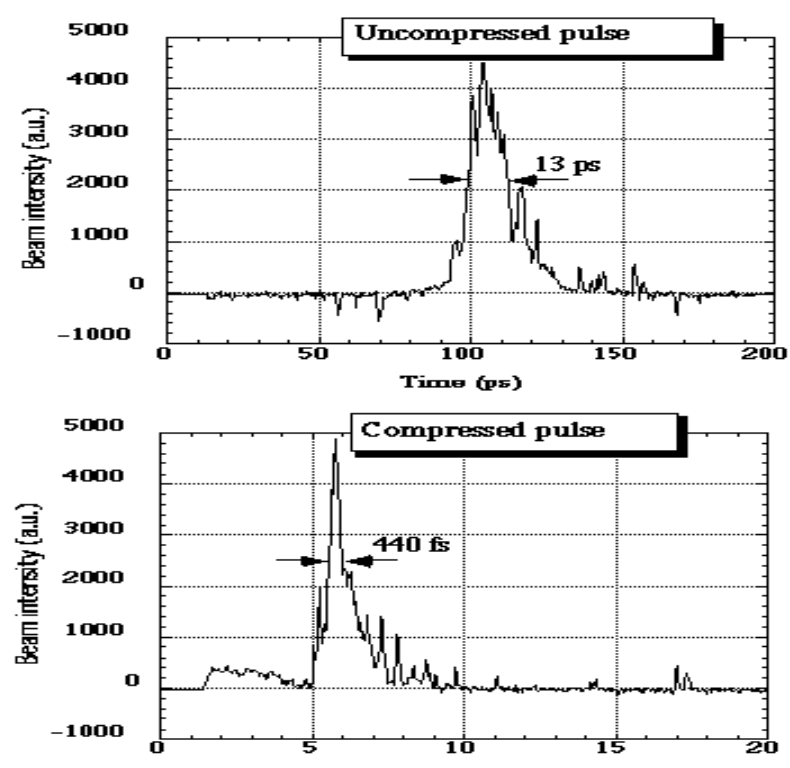

Fig.12 Electron beam bunch length measurements using streak camera [16].

As electron beam bunch length getting shorter, the coherent radiation becomes a viable option. Coherent synchrotron radiation [17], transition radiation [18] and diffraction radiation [19] have been used for bunch length measurements. This technique has demonstrated resolution on the order of 100 fs. The major drawbacks for such technique are multi-shot, precision optical alignment, no self -calibration; bunch distribution information depends on the assumption [20]. Coherent radiation intensity could also be used directly for tuning up the bunch compressor.

In the remaining part of this section, we will focus on two types of technique that is especially suitable for single -pass high-gain FEL. One is phase space reconstruction using tomography; the other is RF based technique, such as slice emittance and RF kicker cavity.

\subsection{Electron Beam Longitudinal Phase Space Tomography}

The longitudinal phase space and bunch length was first measured using tomography technique by the Standford group [21]. The principle is fairly simple, the longitudinal phase of electron beam can be reconstructed using tomography technique by measuring the beam energy spread in the dispersion region as function of the RF linac phase. The compressor and linac combination (Fig.11), which is avail able in single -pass high-gain FEL will be able to rotate the electron beam longitudinal phase space. A special case is to rotate the phase space by 90 degree, so the energy spectrum now is the directly the electron beam bunch length measurement [22]. Fig ure 12 is the photoelectron beam longitudinal phase reconstructed using the tomopgraphy technique [23]. Tomography technique has demonstrated good resolution, give the detailed beam longitudinal distribution information without pre -assumption, and simple to implement for single -pass high-gain FEL because the compressor and linac are existing equipments.

\subsection{RF Base Technique and RF Kicker cavity}

$\mathrm{RF}$ based technique always play an important role in bunch length measurement. We will first review the slice emittance measurement technique using RF phase introduced 
energy chirp. We will then discuss in detail using RF kicker cavity for bunch length measurement.

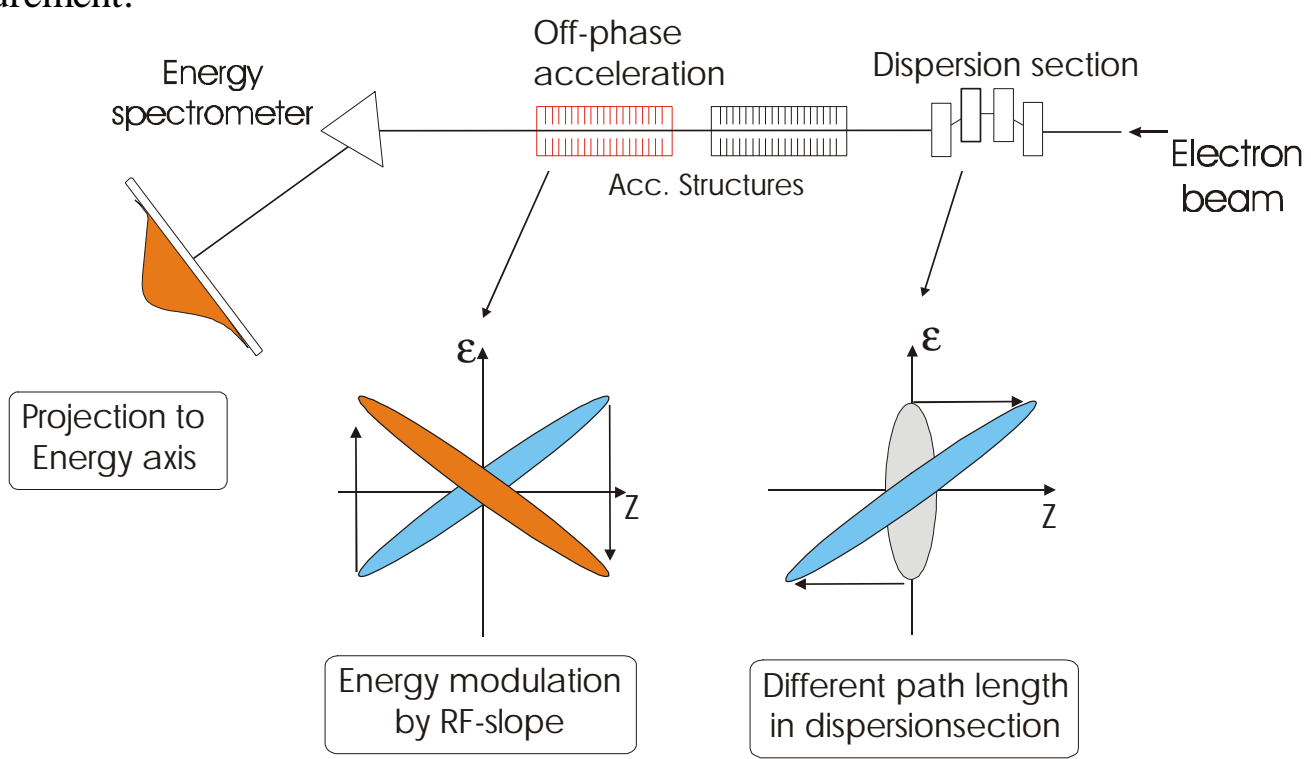

Fig.13 Principle of longitudinal tomography.

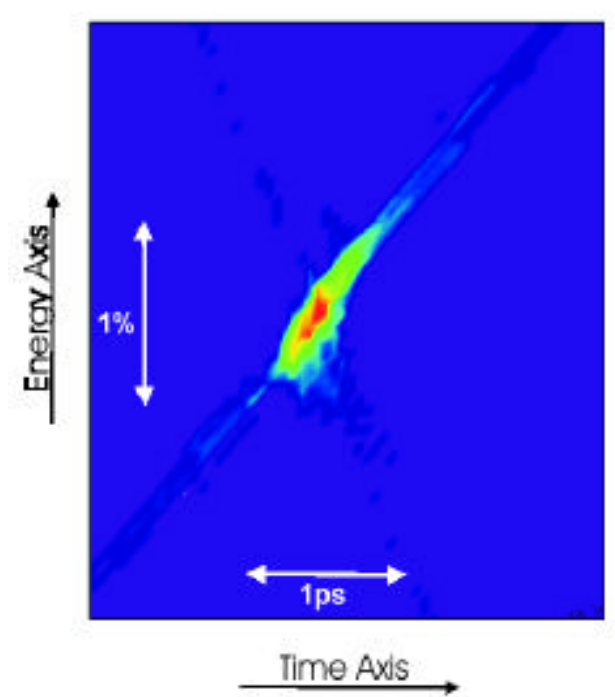

Fig.14 Photoelectron beam longitudinal phase reconstructed by tomography technique.

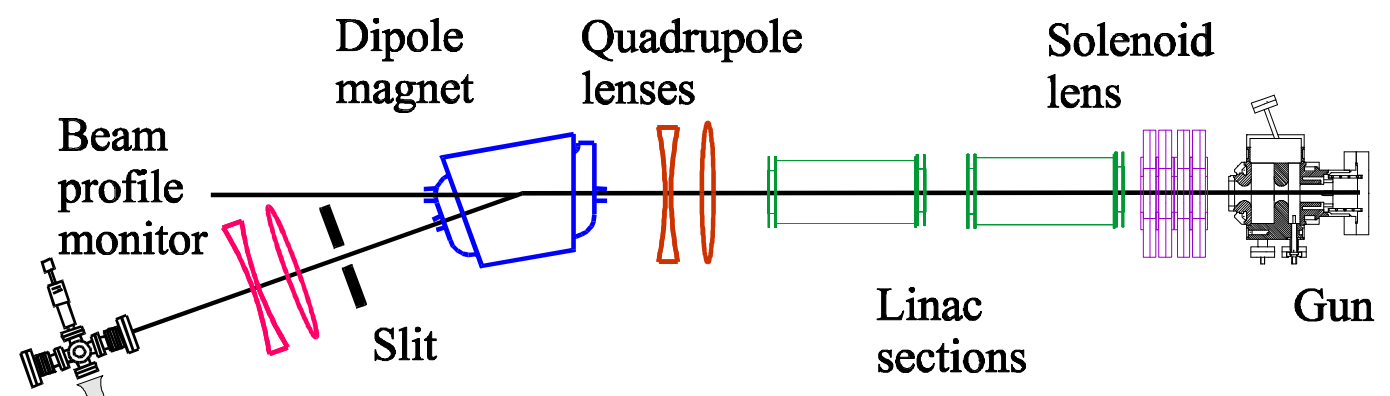

Fig.15 The ATF pico-second slice emittance and bunch length measurements setup. 


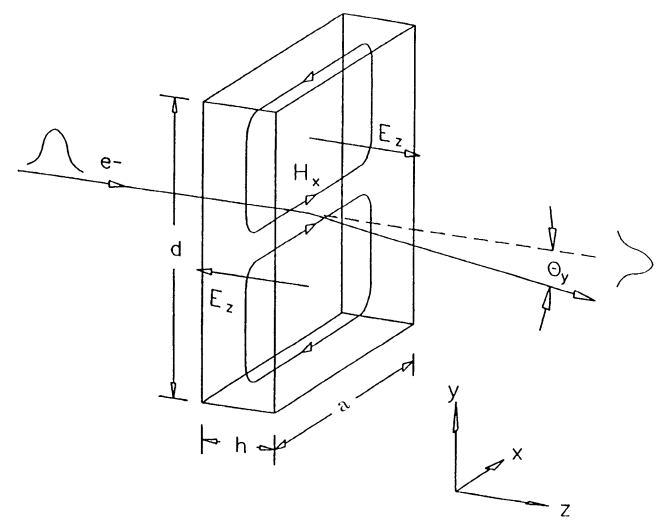

Fig.16 RF kicker cavity schematic.

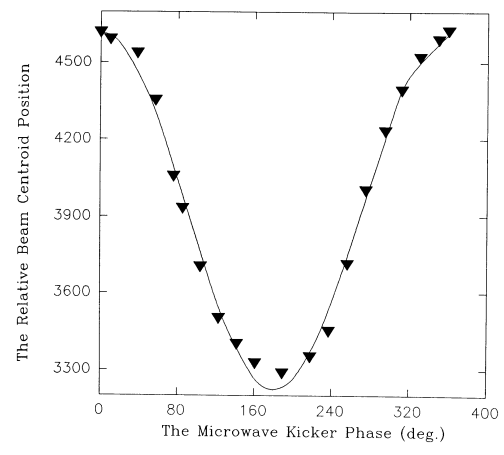

Fig.17 RF kicker calibrat ion curve.

One of the commonly used RF technique for measuring the bunch length is measure the electron beam energy spread in a dispersion region, and electron beam bunch length information is extracted using the relation between the energy spread and $b$ unch length [24]. A Linac RF phase energy chirped technique with energy slit is developed at the ATF with sub-picosecond resolution (figure 15). The electron beam energy is linearly chirped by varying the second section of the linac phase, pico -second slice of electron bunch could be selected by the energy slit positioned after the dipole magnet for both slice emittance [25] and bunch length measurements [26]. Using RF phase energy chirped has the advantage of self-calibration and large dynamic range.

The RF kicker cavity was used for pico-second electron beam characterization at relative low energy [27] (Fig.16). RF kicker cavity application for linear collider and single-pass high-gain X-ray FEL was proposed recently [10] for multi-GeV femtosecond electron beams. The principle of RF kicker cavity for bunch length measurement is similar to that of streak camera, which converts the time information to spatial

$$
\begin{aligned}
& \frac{\Delta p_{y}}{p_{z}} \approx k \Delta z \\
& \frac{\Delta p_{z}}{p_{z}} \approx k y
\end{aligned}
$$

information. One of the common misunderstandings about RF kicker cavity is its applicability to higher energy beam. The RF kicker effect can be written, where $y, z$ are electron vertical and longitudinal coordinates within the bunch, and the kicker strength $k$ is,

$$
k=\frac{2 \pi}{\lambda} \frac{a}{\gamma} \sqrt{P Q}
$$

where $a$ is a constant related to the cavity geometry, $P$ is the RF power, $Q$ is the quality factor of the cavity, $\lambda$ is the RF wavelength, and $\gamma$ is the relativistic factor. Eq. 6 shows that, the scaling for the kicker effect is linearly inverse proportional to the beam energy and RF wavelength. The equation did not take into consideration is the emittance 
decreases with the increase beam energy, so the net kicker effect scales as the inverse square root of the energy. One of the fundamental limitations of the streak camera, space charge effect, is absent for RF kicker cavity. Fu rther more, RF kicker is synchronized with electron beam, and it can be calibrated by measuring the centroid of the beam as function of the RF kicker cavity phase (Fig.17). It can also be used for both the slice emittance and longitudinal phase space mea surements. Using a 2 -meter long S-band traveling RF kicker structure for a $14 \mathrm{GeV}$ LCLS beam, 10 to 20 femto-second resolution can be realized [28].

\section{BEAM DIAGNOSTICS FOR THE UNDULATOR}

A long strong-focusing undulator is required for single -pass high gain FEL to reach saturation, undulator on the order of $100 \mathrm{~m}$ long is now under consideration for the X-ray FEL projects. Beam diagnostics should be considered as a integral part of the undulator design, and it can be divided into two parts. The first part involves measuring the electron beam properties delivered to the undulator, and matching the beam to the undulator. The second part of the beam diagnostics is to measure both electron beam and radiation beam along the undulator, the most challenging iss ue here is to overlap the electron beam and radiation over the long undulator. The local and global alignment required without significant gain degradation is $20 \%$ rms beam size over one gain length, and about rms beam size over whole undulator, that is $5 \mu \mathrm{m}$ for $10 \mathrm{~m}$, and $50 \mu \mathrm{m}$ over 100 meter for the LCLS undulator.

Both longitudinal and transverse electron beam emittance should be characterized in front of the undulator. The bunch length and longitudinal phase space measurement techniques were discusse $d$ in the previous section, we will discuss emittance and beam profile measurement techniques here. Figure 19 is the schematic of experiment setup at the ATF for comparing four different beam profile measuring techniques, optical transition radiation (OTR), scanning wire, phosphor and YAG:Ce screens [29]. The measured resolution for both phosphor and Yag screens is about $100 \mu \mathrm{m}$. The resolution of $30 \mu \mathrm{m}$ for OTR was achieved. The measured resolution for the wire scanner with 25 $\mu \mathrm{m}$ wire is slightly worse tha n OTR because of the jittering of photoelectron beam. The transverse emittance of the electron beam can be measured in several ways. One of the technique successfully employed at the SLAC SLC is to use four beam profile monitors separated by a phase advance $45^{\circ}$ [30](fig.24). This technique is insensitive to the beam matching condition.

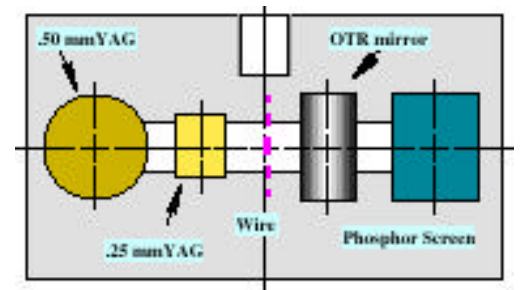

Fig.18 BPM resolution measurements at the ATF.

The emittance measurement using three profile monitors or quadrupole magnet scan are comparable both in resoluti on requirements for the BPM, and the drift distance between quadruple magnet and BPM [31]. We will discuss a combination of quadrupole scan and two beam profile monitor (BPM) for emittance measurement. Generally speaking, two 


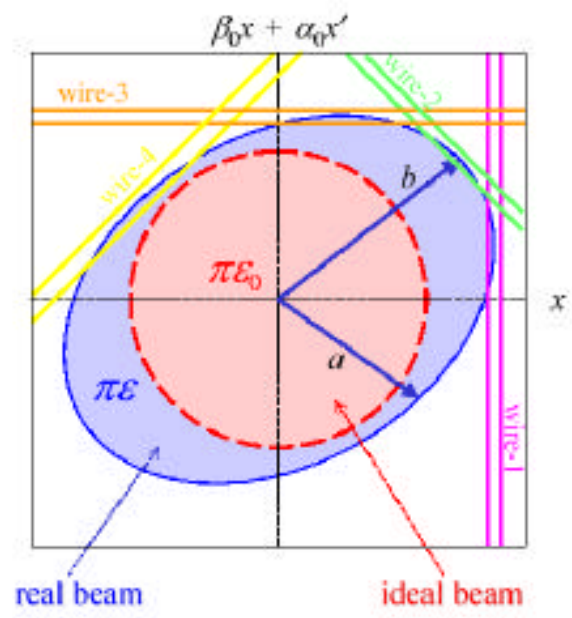

Fig.19 Phase diagram of emitt ance measurement using 4 wire scanners (BPMs). profile monitors do not produce enough information for emittance measurement unless one of the monitors located at the beam waist. This is a rather restricted condition. But if one of the monitor location close to the quadrupole magnet, the minimum spot $x_{\text {min }}$ obtained on the other monitor can be written as:

$$
x_{\min }=\frac{L \varepsilon}{x_{l e n}}
$$

where $L$ is the drift distance between the quadrupole magnet and the BPM, $x_{l e n}$ is the spot size at the qaudrupole. Using above equation, the beam emittance be easily calculated. This arrangement has several advantages. It is simple to implement, and the monitor near the quadrupole magnet could also be used for the beam matching. It can also be used for cross-check with the quadrupole scan .
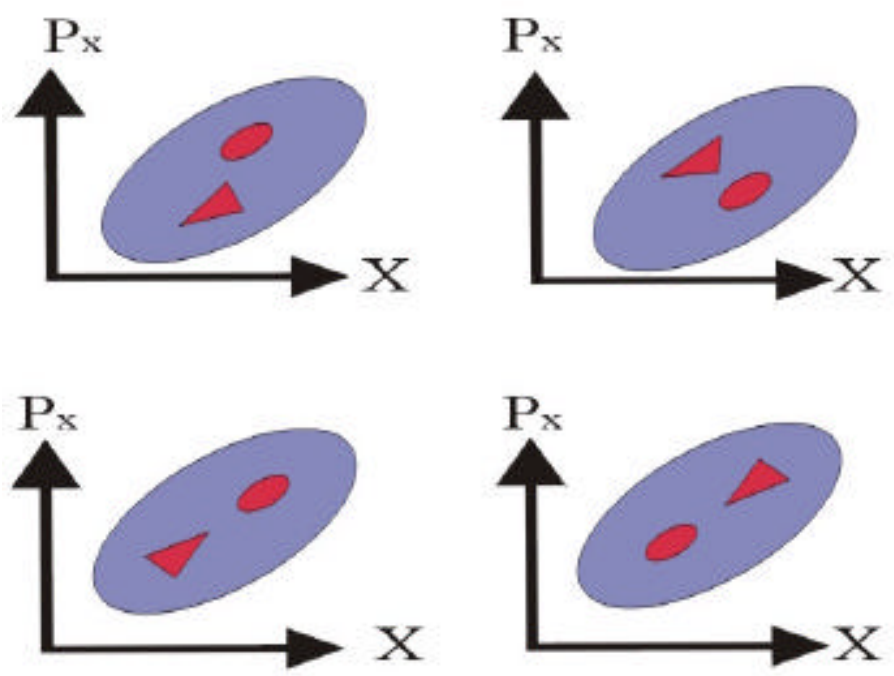

Rotation in phase space is done by changing the currents in many quadrupoles

Fig.20 Schematic transverse phase space tomography. 
Tomography technique was used to reconstruct electron beam phase space[32]. The transverse phase space and emittance are reconstructed by measuring the real space projections of the transverse phase space as it is being rotated by varying the quadruple magnets (Figure 20). Phase space reconstruction using tomopraphy not only provide the emittance information, it also produces the detailed distribution which is needed for emittance growth and FEL studies.

The best beam diagnostics for the long undulator is the FEL radiation itself. Eq.(1) shows that, the gain of the FEL is a strong function of the electron beam peak current and emittance. Optimize the gain of the FEL is one way to improve the electron beam quality. Spontaneous emission from the undulator can also be used for electron beam diagnostics. In the visible range, narrow band of the off -axis radiation from the undulator was used for electron beam energy spread, alignment and emittance characterization [33]. The single-shot fluctuation spectrum was used for the electron beam bunch length measurement [34].

Most single-pass high gain FEL undulators are made up by several segments, each segment length is on the order of 2 to 5 meters. A beam diagnostic station positioned between the segments, figure 21 is the beam diagnostic station for APS LEUTL SASE FEL[4]. Electron beam diagnostics include YAG and optical transition radiation (OTR) screens viewed by a CCD camera. Following the primar y electron beam diagnostics at each station are capacitive pickup beam position monitors with single -shot resolutions of less than $10 \mu \mathrm{m}$ for a bunch charges of $1 \mathrm{nC}$. One of the unique feature of the LEUTL diagnostic station is the undulator radiation meas urement capabilities, so called in -tunnel visible light detectors (VLDs), which consist of additional three -position actuators. These are used to deflect the generated light through a set of filters to a separate CCD camera or to radiation diagnostics room for spectrum and bunch length measurements. The actuator holds an aluminized mirror. The three positions are as follows: 1) out, 2)mirror, 3) mirror with $600-\mu \mathrm{m}$ hole for alignment.

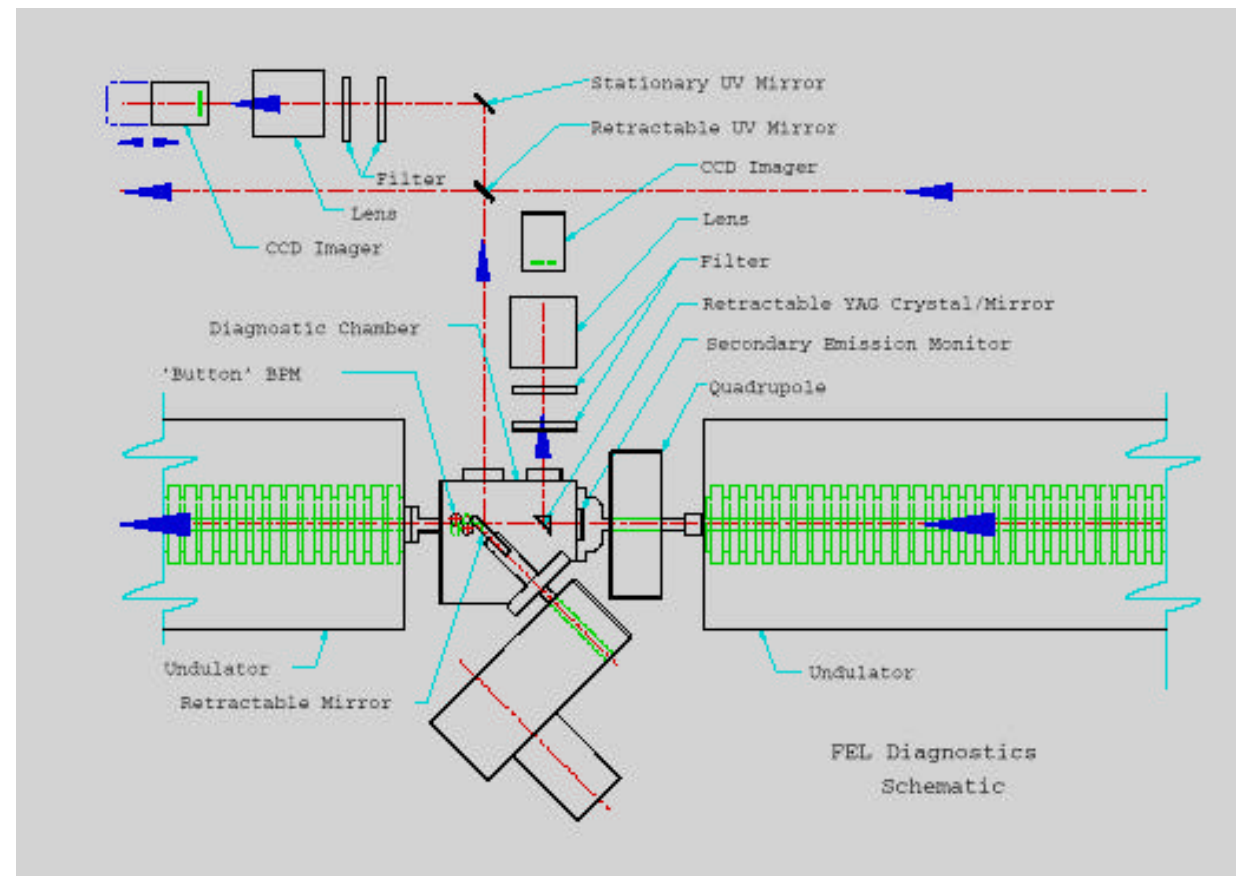

Fig.21 APS LEUTL diagnostic station. 
As we pointed out earlier, the most challenging issue for undulator diagnostic is to aligned the electron beam with the radiation in microns accuracy over 10s meters distance. Most beam based al ignment techniques using beam position monitors [35 -37] as local reference without considering radiation. One of the major differences between linac transport line alignment and single -pass high-gain FEL undulator is that; linac and transport line beam ba sed alignment is relative alignment, while undulator require electron move along the straight line is absolute alignment. Using either YAG or phosphor screens beam profile monitors, both electron beam and radiation can be aligned relative to a reference la ser. Using laser for electron beam trajectories has many advantages. The main limitation is the spot size due to the diffraction. The ideal spot size for a Gaussian beam is,

$$
w=w_{0} \sqrt{1+\left(\frac{\lambda z}{\pi w_{0}^{2}}\right)^{2}} \Rightarrow w_{\min }=\sqrt{\frac{2 \lambda z}{\pi}}
$$

where $z$ is the distance from the beam waist, and $\lambda$ is the laser wavelength. Using $400 \mathrm{~nm}$ blue diode laser, for $z=7$ meter, the minimum spot $w_{\min }$ is about $1.4 \mathrm{~mm}$. So each laser can only cover about $15 \mathrm{~m}$ long segment of the undulator without significantly change the spot size. To cover the whole length of the undulator, several laser alignment stations are needed. Figure 22 is the schematic of the laser alignment station, and each laser is aligned to external common reference lines by periscopes (Figure 23). Each laser station contains two motorized mirrors for remote laser alignment. The optical flats are used for both parallel displacement of the laser beam in both $\mathrm{x}$ and $\mathrm{y}$ directions and calibration purpose. Fig. 24 is a $780 \mathrm{~nm}$ diode alignment laser image for VISA experiment. The stability of the laser is about $20 \mu \mathrm{m}$ over 8 hours. The main issue is the quality of the laser beam image. The image distortion came from several sources, such as vacuum windows and defects on the optics. Using AR coated vacuum windows and adaptive optics technique; the alignment 1 aser image could be improved significantly.

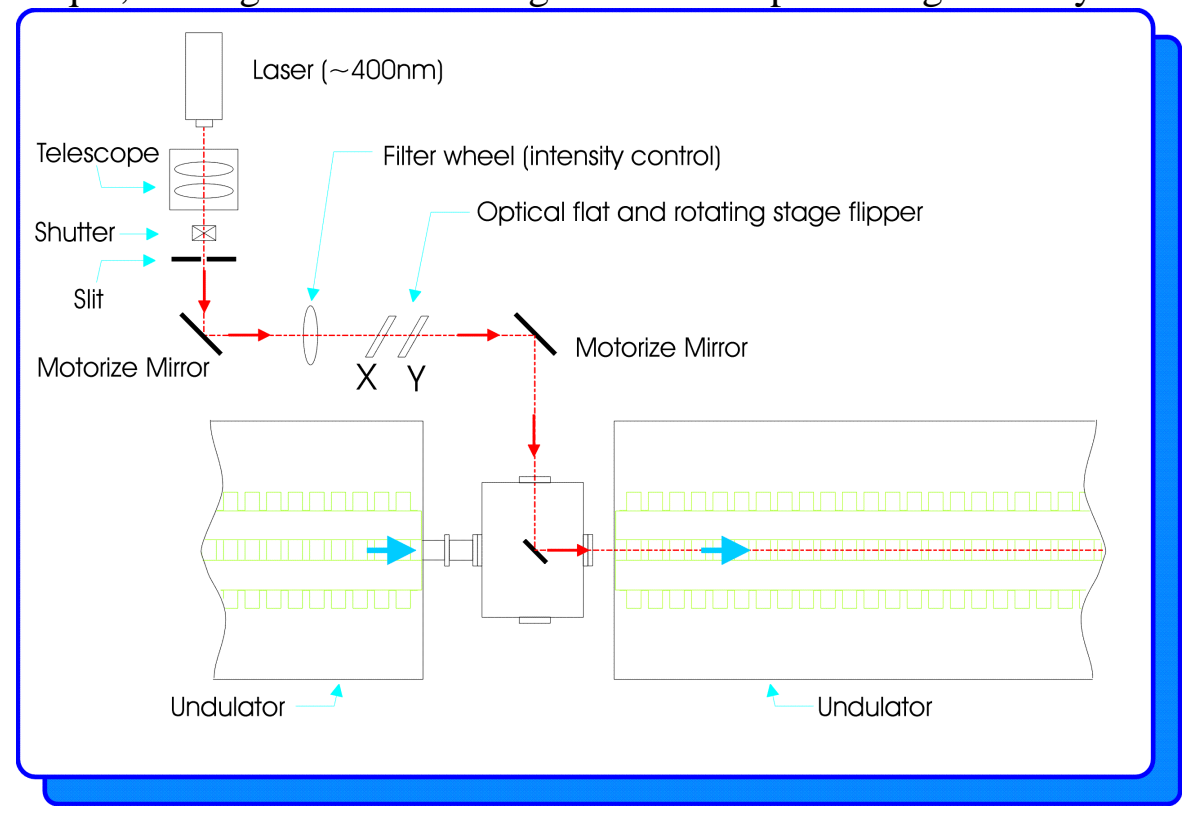

Fig.22 Alignment laser system. 


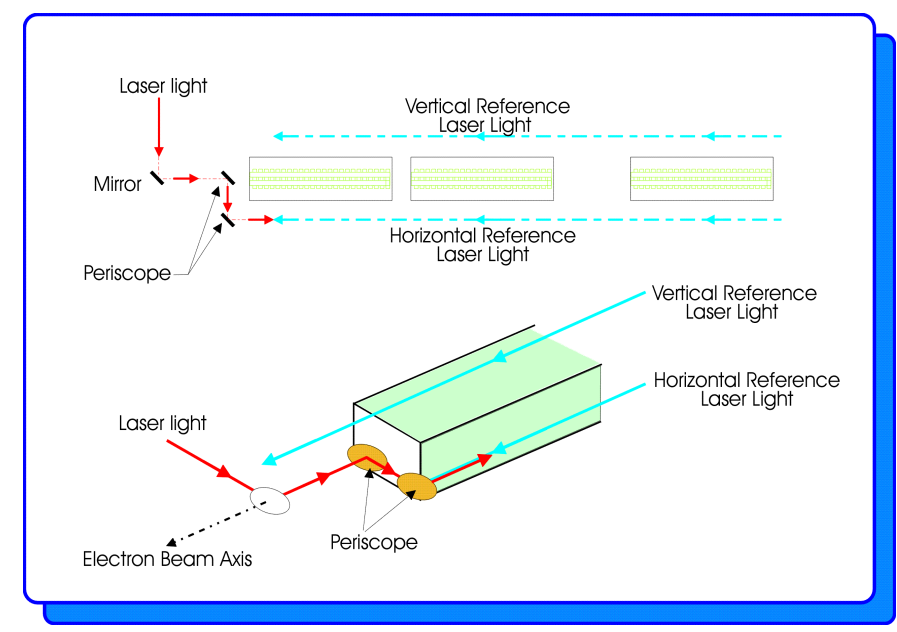

Fig.23 Alignment schematic for multiple laser systems.

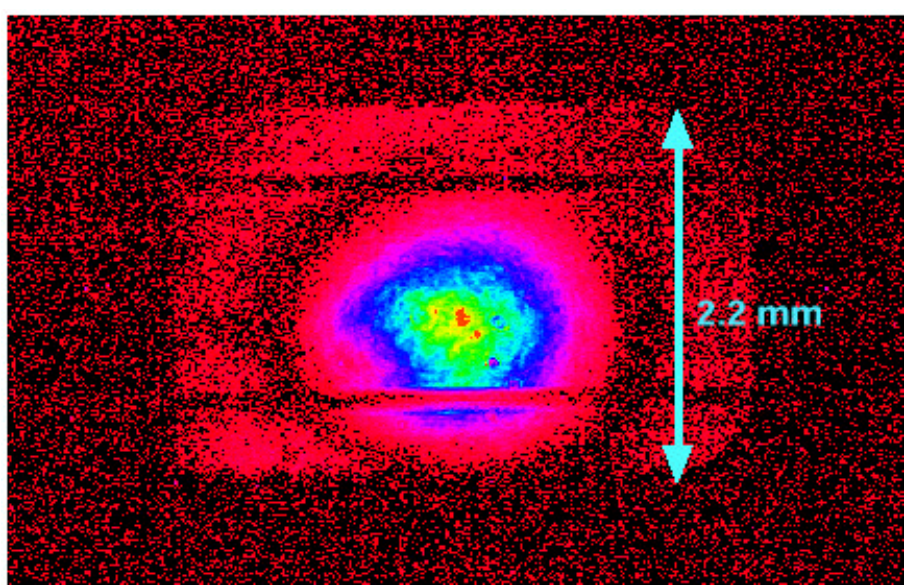

Fig.24 The VISA alignment laser image.

\section{ACKNOWLEDGEMENT}

The author has been benefited greatly from discussions with man y colleagues on the subjects presented here, particularly M. Babzien, I. Ben -Zvi, E. Johson, S. Krinsky, K. Shigruk, V. Yakimenko and L.H. Yu of BNL; S.V. Milton, E. Gluskin, G. Decker, A.H. Lumpkin of APS; M. Cornacchia and P. Emma of SLAC; Aaron Trema ine and Alex Murokh of UCLA; and M. Uesaka of University of Tokyo.

\section{REFERENCES}

1. L.H. Yu et al, "High Gain Harmonic Generation Free Electron Laser", Accepted for publication in Science.

2. R. Bonifacio, C. Pellegrini, L.M. Narducci, Opt. Commun. 50373 (198 4).

3. A. Tremaine et al, "Status and Initial Commissioning of a High Gain $800 \mathrm{~nm}$ SASE FEL", FEL' 99, Hamburg, Germany.

4. S. Milton et al, "FEL development at the APS", Free-Electron Laser Challenges II, Proceeding of SPIE Vol. 3614, San Jose, California (1999) 86.

5. DESY Report TESLA-FEL 95-03, June, 1995.

6. DUV FEL Concept Design Rep., BNL - 48713, Jan., 1994.

7. M. Cornacchia, "The LCLS X-Ray FEL at SLAC", ", FreeElectron Laser Challenges II, Proceeding of SPIE Vol. 3614, San Jose, California (1999) 109. 
8. A.H. Lumpkin, "Overview of Bunch Length Measurements", Free-Electron Laser Challenges II, Proceeding of SPIE Vol. 3614, San Jose, California (1999) 44.

9. G. Neil, "Summary of Working Group VII", ICFA workshop on Future Light Source, http://www.aps.anl.gov/conferences/FLSworkshop/.

10. X.J. Wang, "Producing and Measuring Small Electron Bunches", PAC' 99, p.229.233.

11. R. Sheffield, “ Photocathode RF gun”, AIP conference proceeding 184 (1992) 1500.

12. X.J. Wang et al, "Chanllenges of Operating a Photocathode RF Gun Injector", Linac' 98, Chicago (1998) 866.

13. X.J. Wang and I. Ben-Zvi, "High-Brightness Electron Beam Diagnostics at the ATF", Proceeding of BIW' 96, AIP Conference Proceeding 390 (1996) 232-239.

14. B.E. Carlsten and T.O. Raubenheimer, Phys. Rev. E 51, 1453 (1995).

15. R. Li, EPAC' 2000, Vienna (2000).

16. M. Uesaka et al, "Femto-Second Electron Beam Generation by S -band Laser Photocathode RF gun and Linac", Proceeding of the 8th AAC workshop, AIP conference proceeding 472 (1998) 908.

17. T. Nakazato et al, Phys. Rev. Lett. 63, (1989) 1245.

18. H.-C. Lihn et al, Phys. Rev. E 53, 6 (1996) 6413.

19. Y. Shibata et al, Phys. Rev. E 52, 6(1995) 6787.

20. T. Watanabe et al, Nucl. Instr. And Meth. A 437 (1999).

21. E.R. Crosson et al, Proceeding of Micro Bunches Workshop, AIP conference proceeding 367 (1995) 397.

22. K.N. Ricci and T. Smith, "Longitudinal electron beam and FEL micro -bunch measurements using off -phase RF acceleration", To be published in Phys. Rev. Special Topics Accelerator \& Beam.

23. S. Kashiwagi et al, "Photoelectron Beam Longitudinal Phase Tomography at the BNL ATF", to be published at the Linac' 2000.

24. D.X. Wang et al, Phys. Rev. E 57, 2(1998) 2283.

25. X. Qiu, K. Batchelor, I. Ben-Zvi and X.J. Wang, Phys. Rev. Lett. 76, (1996) 3723.

26. X.J. Wang, X. Qiu and I. Ben-Zvi, Phys. Rev. E. 54 (1996) R3121.

27. X.J. Wang et al, Nucl. Instr. And Meth. A 356 (1995)159-166.

28. P. Emma et al, to be published.

29. A. Murokh et al, "Limitations on the Resolution of YAG:CE Beam Profile Monitor for High Brightness Electron Beam" to be published in World Scientific (2000).

30. P. Emma, "LCLS Beam Instrumentation Requirements", presentation at the LCLS Beam Instrumentation Workshop, BNL, March 2000.

31. K.D. Jacobs et al, "Emittance Measurements at the Bates Linac", 1989 Part. Accel. Conf., p. 1526-1528.

32. V. Yakimenko et al, "Emittance Control of A Beam by Shaping the Transverse Charge Distribution Using a Tomography Diagnostics", EPAC' 98, p.1641- 1643.

33. X. Qiu et al, "Experiments in Non-Perturbative Electron Beam Characterization with MIT Micro-wiggler at the BNL ATF" FEL' 96, BNL63687 (1996).

34. P. Catravas et al, Phys. Rev. Lett. 82 (1999) 5261.

35. J. Safranek, "Beam Based Lattice and Instrumentation Caliberation and Alignment for Storage ring and Transport System", this proceeding.

36. K. Flottmann et al, "Beam Based Alignment Procedure for an Undulator with Superimposed FODO Lattice", TESLA-FEL 97-05, Dec., 1997.

37. P. Emma et al, Nucl. Instr. And Meth. A 429 (1999) 407. 